\title{
Follicular Dendritic Cell Sarcoma of the Larynx: Apropos a Rare Case with Review of the Literature
}

\author{
Ayushi SAHAY, Munita BAL, Asawari PATIL, Shubhada KANE, Prathamesh PAI
}

Department of Pathology, Tata Memorial Center, MUMBAI, MAHARASHTRA, INDIA

\begin{abstract}
Extranodal follicular dendritic cell sarcomas are extremely rare neoplasms included under the hematolymphoid and dendritic group of neoplasms. Histologically, fascicular, whorled spindle cells with sprinkled lymphocytes are typical; however higher grade epithelioid morphology seen in a subset makes diagnosis of follicular dendritic cell sarcoma challenging. Rarity of occurrence, a wide histologic spectrum and a long list of differential diagnostic entities render follicular dendritic cell sarcoma an under-recognized entity susceptible to diagnostic pitfalls. Application of a judicious panel of immunohistochemical markers is imperative to avoid errors in diagnosis and patient management. We report here an extremely rare case of follicular dendritic cell sarcoma of the larynx- a hitherto unreported site.
\end{abstract}

Key Words: Follicular dendritic cell sarcoma, Larynx neoplasm, Podoplanin, Immunohistochemistry

\section{INTRODUCTION}

Follicular dendritic cell sarcoma (FDCS), first reported in 1986 by Monda et al. is a rare tumor that arises from the follicular dendritic cells (1). Principally recognized as a lymph nodal disease, extremely rare occurrences of extranodal FDCS's are reported in the liver, lung, spleen, soft tissues, mediastinum, gastrointestinal tract, etc. Involvement of the head and neck region is extremely uncommon with only few case reports and small series. The tonsil is the most commonly reported site of involvement in the head and neck region (2-4). Spindle to epithelioid cells, intimately admixed with lymphocytes, is the typical histology. However the morphologic spectrum of FDCS is broad, inviting diverse clinicopathologic entities into the list of differential diagnosis.

Extreme rarity at extranodal sites coupled with lack of awareness of its varied morphologic features makes diagnosis of FDCS challenging and fraught with pitfalls. Herein, we report a case of FDCS arising in the larynx, a heretofore unreported site till date.

\section{CASE REPORT}

A 59-year-old male patient presented with a history of swelling on the left side of the neck for eight months, hoarseness of voice for three months, dysphagia for one month, and mild breathlessness. The patient had undergone treatment for pulmonary tuberculosis 25 years back. He gave a history of smoking one packet per day of beedi (a

(Turk Patoloji Derg 2019, 35:254-257)

Received : 18.03.2017 Accepted : 30.06.2017 thin Indian cigarette, filled with tobacco flakes and rolled in leaf) since 18 years of age.

On examination, bilateral rhonchi were noted. Left level III cervical lymph node was enlarged and cystic. On direct laryngoscopy, there was a large proliferative growth involving epiglottis, bilateral aryepiglottic folds, bilateral pharyngoepiglottic folds, bilateral vallecula, bilateral false vocal cords and left pyriform sinus. The glottic chink was compromised. The right pyriform sinus, and post cricoid area were free. The right vocal cord was mobile.

On chest X-Ray, ill-defined patchy opacities were seen in right upper and left mid zone. Few fibrocalcific opacities were also seen, suggestive of post-tubercular fibrosis with cavitation. On computerized tomography (CT) scan, a mass was seen involving the posterior $1 / 3^{\text {rd }}$ of the tongue, vallecula, epiglottis, and both sides of the hypopharynx and supraglottis. The pre-epiglottic space was effaced. The mass was crossing the midline and involving the lateral hypopharyngeal wall, and invading the prevertebral muscles at the C4-C5 level. No cartilage erosion was seen. A large necrotic lymph node was noted at left level III. The patient underwent a laryngoscopic biopsy from the supraglottis. Subsequently, the patient developed aspiration and breathlessness for which he underwent an emergency tracheostomy.

Microscopic examination of the laryngoscopic biopsy revealed an overlying partly ulcerated squamous mucosa

Correspondence: Munita BAL

Department of Pathology, Tata Memorial Center,

MUMBAI, MAHARASHTRA, INDIA

E-mail: munitamenon@gmail.com Phone: +9122 24177000 
with a subepithelial tumor, composed of large, pleomorphic epithelioid neoplastic cells with rounded nuclei, pale chromatin, distinct nucleoli and abundant pale eosinophilic cytoplasm (Figure 1A). The tumor tended to have a syncytial appearance attributable to indistinct cytoplasmic borders. A conspicuous sprinkling of small lymphocytes throughout the tumor was present. Frequent mitoses (1520/10 high power fields), including atypical mitotic figures, were seen (Figure 1B). Areas of necrosis were identified focally. On immunohistochemical examination, the tumor was strongly immunoreactive for CD23 (Figure 1C), and D2-40 (podoplanin) (Figure 1D) and immunonegative for AE1/AE3, CD21, p40, p63, LCA, CD3, CD20, CD30, S100, CD68, CD1a, EBV LMP-1 and CD34.
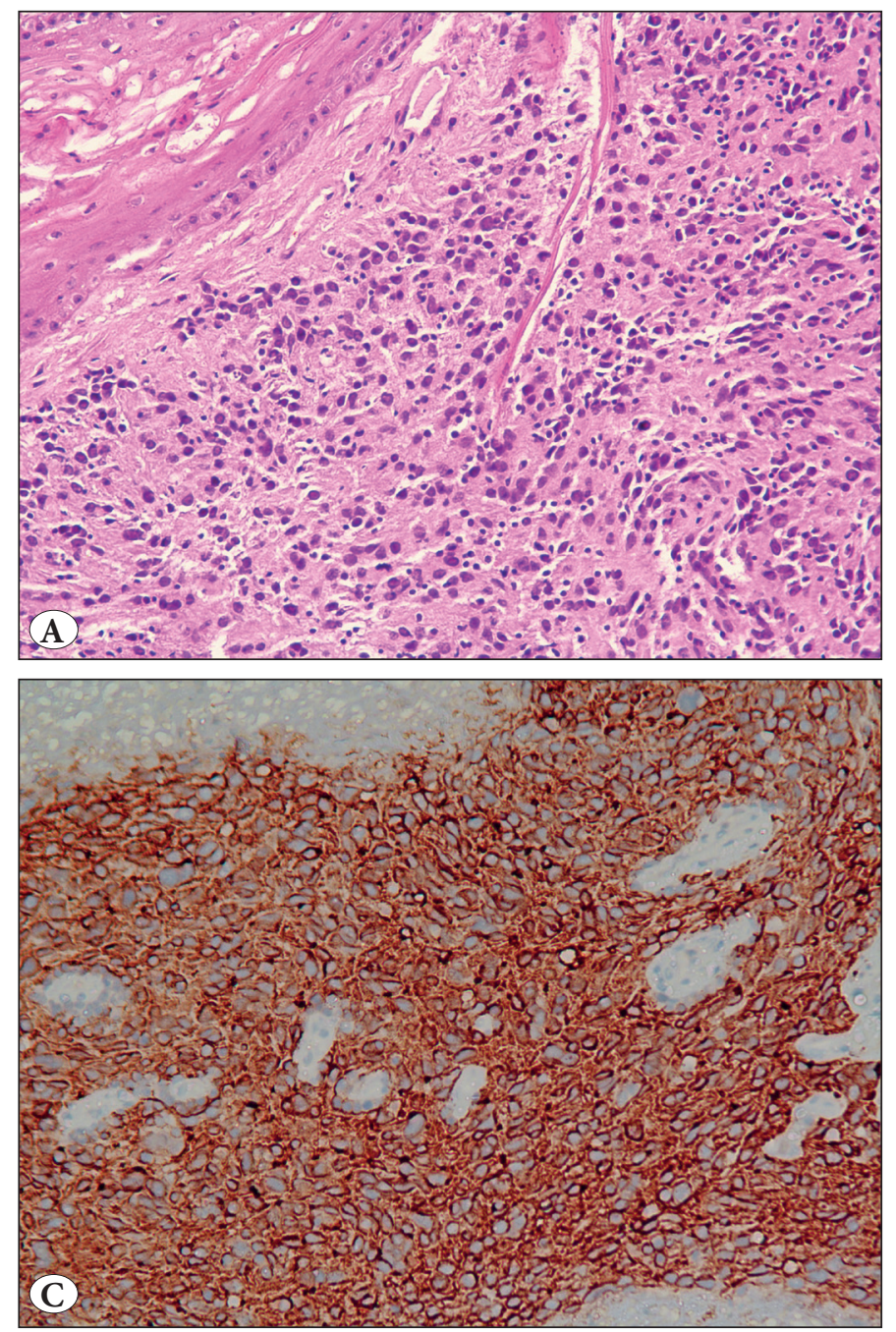

In view of the morphological and immunohistochemical features, a diagnosis of high grade follicular dendritic cell sarcoma involving the larynx was proffered.

Due to involvement of the vallecula, the mass was considered unresectable, hence chemotherapy followed by reassessment was planned. However, the patient refused further treatment and was subsequently lost to follow-up.

\section{DISCUSSION}

Follicular dendritic cells function primarily as antigen presenting cells to B cells, hence are involved in humoral immunity $(2,5)$. Tumors arising from follicular dendritic cells are called follicular dendritic cell sarcoma, and are classified under the histiocytic and dendritic cell neoplasms

Figure 1: A) Laryngeal biopsy showing squamous epithelium with underlying diffuse sheets of tumor cells (H\&E; x100). B) High magnification showing large epithelioid cells with abundant pale eosinophilic cytoplasm, marked pleomorphism, high grade nuclei with hyperchromasia. Lymphocytes are sprinkled amidst neoplastic cells. A mitotic figure is seen (arrow) (H\&E; x400). C) Strong immunohistochemical membranous positivity for CD23 (IHC; x400). D) Strong immunohistochemical membranous positivity for D240 (podoplanin) (IHC; x400). 
in the World Health Organization classification of tumors (6).

Etiopathogenetic factors of FDCS's are yet to be clearly defined. In view of some cases arising in the background of Castleman disease, a hyperplasia-dysplasia-neoplasia sequence, akin to colorectal cancer, has been proposed for FDCS's $(2,4)$. Other possible etiological factors proposed are EBV infection, possible association with autoimmune disorders, and origin from a common multipotent mesenchymal stem cell $(2,4,6)$.

In a pooled analysis of cases reported from 1978-2012, Saygin et al. found 343 cases of FDCS's in the literature, with a mean age at diagnosis being 50 years, and an almost equal male-female predilection (4). Though initially considered a nodal disease, they observed that FDCS was increasingly being reported at extranodal sites, including liver, spleen, soft tissues, mediastinum, gastro-intestinal tract, mesentery, bone, pancreas, and skin. Other rare sites, reported as isolated cases, include the kidney, pleura, testis, ovary, bladder, adrenal, dura, and thymus (4). Recently, Pang et al. reviewed English literature specifically for head and neck region FDCS and found 97 cases, most commonly involving the lymph nodes (40\%), oropharynx (24\%) and soft tissues of the head and neck (10\%) (7). In a larger review, Li et al. analyzed 137 cases of head and neck FDCS, with an equal male to female distribution, similar to FDCS elsewhere in the body (8). The mean age at diagnosis was 46 years (9-79 years). Of the 137 cases, 65 were cervical nodal FDCS and 72 were extranodal. The most common extranodal site was the tonsil, followed by the nasopharynx, parapharyngeal space, palate, thyroid and parotid (8). However, involvement of the larynx by FDCS, as seen in the present case, has not been reported till date, to the best of our knowledge.

Clinically, the majority of the cases present as slowly growing painless masses, which on gross appearance are usually well circumscribed and solid (4). Microscopically, FDCS's are composed of neoplastic follicular dendritic cells intimately admixed with lymphocytes. Two types of histological patterns are recognized (9). In the low grade pattern, the tumor cells are uniform, spindle to ovoid, arranged in short fascicles, whorls, or nodules. Nuclei show mild atypia, pale chromatin, distinct nuclear membrane and visible nucleoli. Hyperchromasia is not typical. The mitotic count is usually low. Admixture with lymphocytes sprinkled throughout the tumor, with or without plasma cells, is seen in more than $90 \%$ of the cases $(4,9)$. Depending on the location, these tumors are often histologically confused with diverse pathologic entities like nerve sheath tumors, gastrointestinal stromal tumors (GIST), inflammatory myofibroblastic tumor (IMT), fibrous histiocytomas, meningiomas, Type A thymoma, and granulomatous inflammation.

In contrast, in the high grade pattern, the tumor cells are arranged predominantly in diffuse sheets, with areas of necrosis. The tumor cells have epithelioid or pleomorphic morphology, with marked nuclear atypia and frequent mitosis. Lymphocytic infiltrate, considered characteristic of FDCS, may be sparse $(4,9)$. High grade FDCS may be mistaken for poorly differentiated/ undifferentiated carcinoma, melanoma, lymphoma, malignant sarcoma, and other histiocytic and dendritic cell neoplasms. In the current case, the tumor resembled a poorly differentiated squamous cell carcinoma (SCC), with epithelioid appearance, areas of necrosis, and frequent mitosis, albeit with a striking admixture of lymphocytes, which served as an important clue, leading us to include FDCS markers in our differential diagnoses. Immunohistochemistry (IHC) is essential for confirming the diagnosis. On IHC, FDCSs are positive for CD21, CD35, CD23, vimentin, desmoplakin, clusterin and fascin. They are variably positive for CD68, EMA and S100 protein (9). MIB-1 labeling indices range from 1-25\% (mean $13 \%)(6,9)$. Recently podoplanin (D2-40), has been shown to be a sensitive marker for FDCS, and was also positive in our case (10). However, not infrequently, staining for many of the FDCS markers is focal. In their review, Li et al. found positivity rates for $\mathrm{CD} 21, \mathrm{CD} 35, \mathrm{CD} 23$, and vimentin to be $85 \%, 55 \%, 31.7 \%$ and $38.3 \%$, respectively (8). Hence it is imperative to use more than one FDCS marker and to repeat the markers on additional blocks when the index of suspicion is high (4). In the present case too, the tumor was strongly immunopositive for both CD23 and podoplanin, however, it was immunonegative for CD21 (possibly because the specimen was a biopsy with limited amount of tumor tissue), highlighting the importance of performing multiple markers for FDCS. Immunohistochemistry for CD35 is not available at our center. These tumors are negative for immunohistochemical markers of the majority of the entities in the differential diagnosis, including cytokeratin, HMB45, CD3, CD20, ALK-1, CD30, CD34, CD79a, lysozyme, CD1a, leucocyte common antigen (LCA), and myeloperoxidase (9).

Extranodal FDCS is still an under-recognized entity, especially in the head and neck. It poses a potential diagnostic pitfall for pathologists, because even though the histological and immunohistochemical features are quite characteristic, it is commonly confused with more common and more aggressive tumors such as squamous carcinoma, sarcomatoid carcinoma, melanoma, lymphoma and 
mesenchymal tumors. Hence, the diagnosis is often missed, especially in a needle biopsy, primarily due to a failure to consider FDCS in the differential diagnosis, and thus not employing an appropriate immunohistochemical panel. Also, the large panel of IHC markers necessitated to rule out other diagnoses may not be available at smaller centers. Because of the wide variety of differentials, and the rarity of FDCS, a stepwise approach eliminating the more common conditions, before proceeding to specific FDCS markers, is generally more cost effective.

FDCSs are considered to be intermediate grade sarcoma because of the substantial risk of local recurrence (23-43\%) and metastasis (21-27\%) with a mortality rate of about $3-7 \%$. In extranodal FDCS, the 2- and 5-year survival rates are only 62.3 and $27.4 \%$ respectively $(4,7)$. Some authors have suggested that features purporting an adverse outcome, similar to other soft tissue sarcomas, include young age at diagnosis, intra-abdominal location, large tumor size $(\geq 6 \mathrm{~cm})$, coagulative necrosis, absence of lymphocytes, significant nuclear pleomorphism and high mitotic count ( $\geq 5 / 10 \mathrm{HPF})(2,4,6,9)$.

Current treatment approach for FDCS at all sites, including the head and neck, is surgical excision with clear margins, with or without adjuvant chemotherapy or radiotherapy (6-8). In advanced or metastatic disease, a combined approach, with diverse modalities, including chemotherapy and radiotherapy, have been attempted (4). In the present case, as the disease was unresectable at presentation and chemotherapeutic intervention was planned; however the patient refused treatment, and was lost to follow up.

In summary, we report the first case of extranodal FDCS arising in the larynx, to the best of our knowledge. Its rarity, wide morphological spectrum and long list of differential diagnostic entities make FDCS an under-recognized disease and susceptible to diagnostic pitfalls. A high index of suspicion together with a judicious use of IHC can help segregate FDCS from other spindle cell or poorly differentiated epithelioid tumors.

\section{CONFLICT of INTEREST}

The authors declare no conflict of interest.

\section{REFERENCES}

1. Monda L, Warnke R, Rosai J. A primary lymph node malignancy with features suggestive of dendritic reticulum cell differentiation. A report of 4 cases. Am J Pathol. 1986;122:562-72.

2. Youens KE, Waugh MS. Extranodal follicular dendritic cell sarcoma. Arch Pathol Lab Med. 2008;132:1683-7.

3. Biddle DA, Ro JY, Yoon GS, Yong YW, Ayala AG, Ordonez NG, Ro J. Extranodal follicular dendritic cell sarcoma of the head and neck region: Three new cases, with a review of the literature. Mod Pathol. 2002;15:50-8.

4. Saygin C, Uzunaslan D, Ozguroglu M, Senocak M, Tuzuner N. Dendritic cell sarcoma: A pooled analysis including 462 cases with presentation of our case series. Crit Rev Oncol Hematol. 2013;88:253-71.

5. Rezk SA, Nathwani BN, Zhao X, Weiss LM. Follicular dendritic cells: Origin, function, and different disease-associated patterns. Hum Pathol. 2013;44:937-50.

6. Chan JKC, Pileri SA, Delsol G, Fletcher CDM, Weiss LM, Grogg KL. Follicular dendritic cell sarcoma. In Swerdlow SH, Campo E, Harris NL, Jaffe ES, Pileri SA, Stein H, Thiele J, Vardiman JW, editors. WHO classification of tumors of hematopoietic and lymphoid tissues. Lyon: IARC; 2008.363-4.

7. Pang J, Mydlarz WK, Gooi Z, Waters KM, Bishop J, Sciubba JJ, Kim YJ, Fakhry C. Follicular dendritic cell sarcoma of the head and neck: Case report, literature review, and pooled analysis of 97 cases. Head Neck.2016;38 Suppl 1:E1501-9.

8. Li J, Zhou ML, Zhou SH. Clinical and pathological features of head and neck follicular dendritic cell sarcoma. Hematology.2015;20:571-83.

9. Soriano AO, Thompson MA, Admirand JH, Fayad LE, Rodriguez AM, Romaguera JE, Hagemeister FB, Pro B. Follicular dendritic cell sarcoma: A report of 14 cases and a review of the literature. Am J Hematol. 2007;82:725-8.

10. Yu H, Gibson JA, Pinkus GS, Hornick JL. Podoplanin (D240 ) is a novel marker for follicular dendritic cell tumors. Am J ClinPathol. 2007;128:776-82. 